\title{
Amphibia-Reptilia \\ Phenology and temperature are the main drivers shaping the detection probability of the common wall lizard \\ --Manuscript Draft--
}

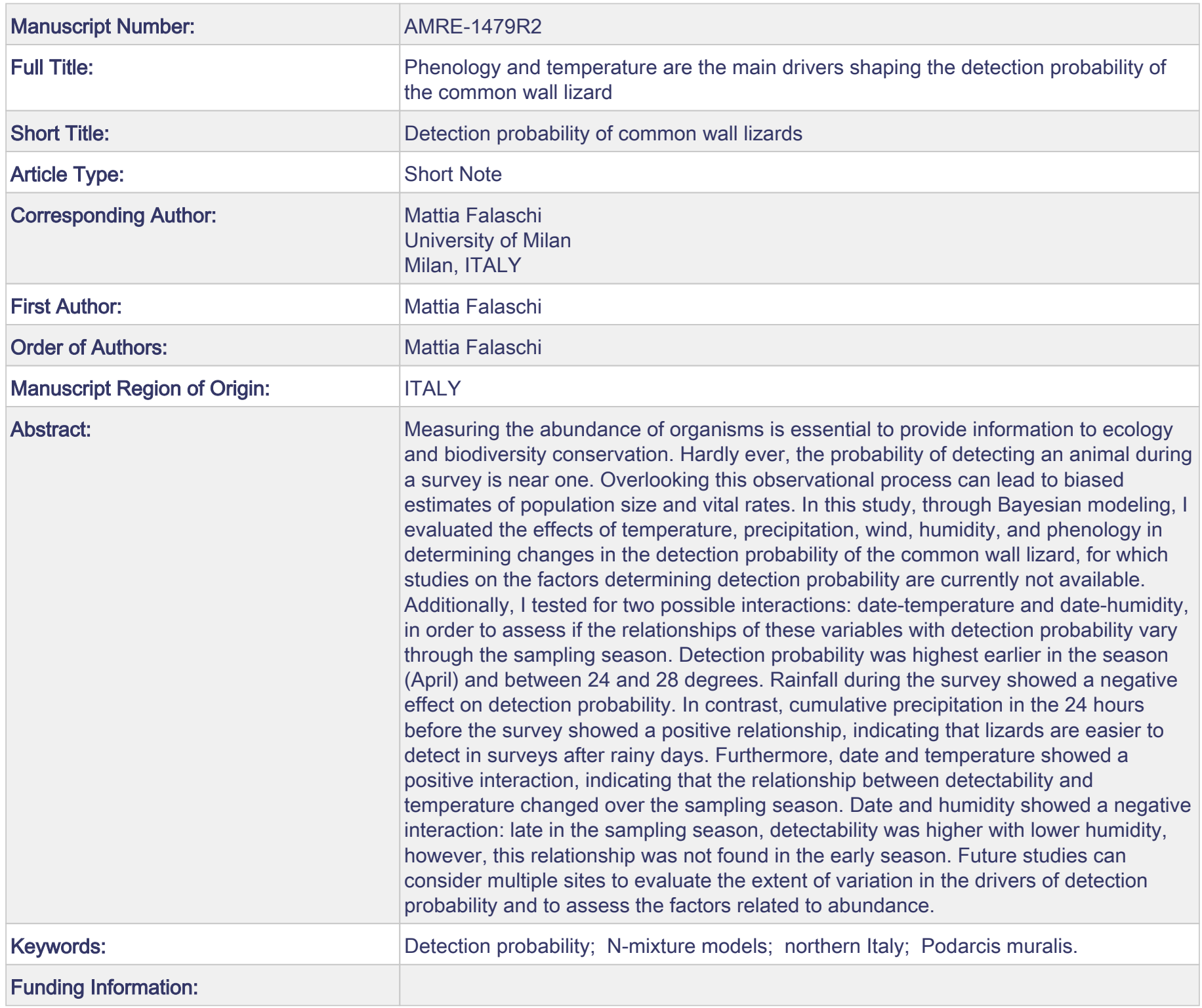




\title{
Dear Dr. José C Brito \\ I revised my manuscript entitled "Phenology and temperature are the main drivers shaping the detection probability of the common wall lizard" following the issue raised by reviewer \#1.
}

I hope that this revised version is now suitable for publication in Amphibia-Reptilia.

\author{
Sincerely, \\ Mattia Falaschi
}

Associate Editor: This is the second version of the manuscript. Reviewer \#1 considers it satisfactory and only raises a minor point. Unfortunately, reviewer \#2 did not reply as also three more potential reviewers. To prevent further delays at this advanced stage of the revision and because in fact this is my area of expertise, I have personally reviewed the text to ensure that the minor amendments suggested by reviewer \#2 were implemented and that was the case point by point. Overall, I consider that authors should only justify the criticism about the rainy days.

Response: I thank the associate editor for the effort to provide a fast review process. I modified the manuscript following the issue about rainy days raised by reviewer \#1.

Reviewer \#1: The author revised the manuscript very well following the comments or suggestions of the reviewers. I am overall satisfied with the revision and their responses to my review comments. However, I still have a concern on the manuscript before it can be considered for publication.

\section{Response: I thank the reviewer for the positive comment.}

My main concern is that the author should inform the reader about the lack of sample in rainy days (only 3 days). In the whole text the author presents precipitation as a main factor that influences detection probability but does not mention that rained only three days during the survey. Although the results obtained with precipitation has a biological sense, I think the author should tone down his conclusion regarding the precipitation, at least making the readers aware of the lack of sample. The results and other previous works supported the negative effect of precipitation in detection 
probability in lizards and although the positive effect in detection probability of the cumulative precipitation $24 \mathrm{~h}$ before is an important factor to really take into account, I think the author should conclude the precipitation section that it is necessary a higher sample or further studies to be comfirmed the interesting results.

Response: I agree with the reviewer's comment about precipitations during the survey. However, for cumulated precipitation in the previous 24 hours, there is more data. While only three surveys were carried out during rainfall $(2.5 \%), 35 \%$ of surveys are associated with values of cumulated precipitation during the previous 24 hours $>0$. For this reason, I added a sentence explaining the lack of data for precipitation during the survey and the reliability of cumulated precipitation in the previous 24 hours (lines 226-229). Given the uncertainty about the relationship between precipitation during survey and detection probability, I changed the plot in Figure $2 b$ to show the relationship between detection and cumulated precipitation and not precipitation during the survey. 
1 Phenology and temperature are the main drivers shaping the detection probability

2 of the common wall lizard

3

$4 \quad$ Mattia Falaschi ${ }^{1, *}$

5

$6{ }^{1}$ Department of Environmental Science and Policy, Università degli Studi di Milano,

$7 \quad$ Via Celoria 10, 20133 Milano, Italy

$8 \quad{ }^{*}$ Corresponding author. E-mail: matt_fala@hotmail.it

9

10 Type of manuscript: Article

11 Manuscript words: 2365

12 Abstract words: 248

13 Short title: Detection probability of common wall lizards 


\section{Abstract}

17 Measuring the abundance of organisms is essential to provide information to ecology and biodiversity conservation. Hardly ever, the probability of detecting an animal during a survey is near one. Overlooking this observational process can lead to biased estimates of population size and vital rates. In this study, through Bayesian modeling, I evaluated the effects of temperature, precipitation, wind, humidity, and phenology in determining changes in the detection probability of the common wall lizard, for which studies on the factors determining detection probability are currently not available. Additionally, I tested for two possible interactions: date-temperature and date-humidity, in order to assess if the relationships of these variables with detection probability vary through the sampling season. Detection probability was highest earlier in the season (April) and between 24 and 28 degrees. Rainfall during the survey showed a negative effect on detection probability. In contrast, cumulative precipitation in the 24 hours before the survey showed a positive relationship, indicating that lizards are easier to detect in surveys after rainy days. Furthermore, date and temperature showed a positive interaction, indicating that the relationship between detectability and temperature changed over the sampling season. Date and humidity showed a negative interaction: late in the sampling season, detectability was higher with lower humidity, however, this relationship was not found in the early season. Future studies can consider multiple sites to evaluate the extent of variation in the drivers of detection probability and to assess the factors related to abundance.

\section{Keywords}


Detection probability, N-mixture models, northern Italy, Podarcis muralis.

\section{Introduction}

Measuring the abundance of organisms is essential to provide information to ecology and biodiversity conservation. While simple counts of population size can be easy to obtain, the probability of detecting an individual during a survey is usually less than one. Imperfect detection can be the results of different factors acting jointly, such as environmental conditions, observer skill, or species traits (Mazerolle et al., 2007;

Kellner and Swihart, 2014). Not including this observational process into models can lead to biased estimates of population size, vital rates such as survival probability, and of relationships with covariates driving these parameters (Kéry and Schaub, 2012). Since the early 2000s, there has been a considerable increase in methods able to include detection probability into models and in their use (MacKenzie et al., 2003; Royle, 2004; Manenti et al., 2020). However, many studies still do not consider imperfect detection, even if this pattern can vary across taxa (Kellner and Swihart, 2014). Species with a cryptic behavior or a cryptic color pattern can be particularly hard to detect, and this is the case for many reptiles (Mazerolle et al., 2007; Ficetola et al., 2018, 2021). Many factors can influence the probability of seeing an individual during a survey. These factors can be either site-specific, such as the vegetation type, surveyspecific, such as weather conditions during the survey, or may depend on individual heterogeneity, such as life-stage or sex. For instance, the activity of ectothermic vertebrates can be strongly influenced by abiotic factors such as temperature, humidity, and precipitation (Daltry et al., 1998; Sun et al., 2001). Another factor that can affect 
activity patterns is phenology. Many species are more active and easier to detect during the breeding season, reducing activity in other periods of the year (Braña, 1991;

Zamora-Camacho et al., 2013). If few surveys are available to assess the status of a species in a certain area, it is best to carry out those surveys when the probability of finding the target species is highest. For this reason, knowing the factors that influence species' detection probability is crucial to optimize the monitoring of both rare and common species.

In this study, I focused on the common wall lizard Podarcis muralis, a lacertid lizard distributed in central and southern Europe (Sillero et al., 2014). Many aspects of the ecology and ethology of this species have been intensively studied, including its polymorphism, aggressive behavior, hematology, and demography (Gracceva et al., 2008; Scali et al., 2016, 2019; Pérez i de Lanuza and Carretero, 2018; Sacchi et al., 2020). However, so far, no study has ever focused on the factors related to detection probability in this species, even if it is a widespread and common reptile. For this reason, I estimated the relative effect of several candidate drivers of detection probability in the common wall lizard. By performing a large number of surveys at a site in northern Italy, I evaluated the effects of temperature, precipitation, wind, and humidity in determining changes in detection probability. Additionally, I considered the effect of the date of the survey to consider the phenology. Furthermore, I tested for two possible interactions: between date and temperature, and between date and humidity, in order to assess if the relationship between these two variables and detectability varied over the sampling season.

\section{Material and methods}


Study area and sampling

89

The study was carried out in Cardano al Campo, Lombardy, northern Italy, coordinates: 45.6367N, 8.7710E. The study site is a residential area composed of roads, houses, private gardens, and meadows (Fig. S1). Walking around the streets, it is easy to spot the common wall lizard, a small lacertid lizard with a maximum snout-length of $\sim 75$ mm (Biaggini et al., 2011), mating, hunting, or basking onto the walls. I performed repeated counts of lizards within this area by walking along a pre-defined path of $\sim 1.1$ $\mathrm{km}$ in length (Fig. S1). The path was walked at a slow speed (between 2 and $3 \mathrm{~km} / \mathrm{h}$ ) to allow a careful inspection of both sides of the roads. A total of 117 surveys were performed between 12 April and 6 October 2020, a period covering the peak of activity of this species (Biaggini et al., 2011). On some days, I carried out two surveys, while in others, no survey was carried out. The average frequency of surveys was one every 1.5 hatched individuals (total length 5-6 cm; Biaggini et al., 2011) were excluded from the analyses.

\section{Environmental data}

Environmental data were gathered from a weather station of the regional agency for the protection of the environment 
station is located near the study site (station coordinates: $45.61924 \mathrm{~N}, 8.75697 \mathrm{E}$ ) and registers weather data every 10 minutes. Temperature and precipitation are two crucial variables shaping reptiles' activity (Zamora-Camacho et al., 2013; Cunningham et al., 2016). Additionally, humidity and wind can be important determinants of activity patterns (Daltry et al., 1998; Sun et al., 2001). Hence, for each survey, I extracted values of mean temperature, mean humidity, mean wind speed, and cumulative precipitation. As the duration of a survey was 25-30 min, weather data values were averaged across the 30 min timespan corresponding to the time when each survey was carried out. Additionally, I calculated the cumulative precipitation in the 24 hours before the survey to test for a possible effect of rainfall on the activity of the following day.

Statistical analyses

$\mathrm{N}$-mixture models can reliably estimate population abundance and detection probability of vertebrates (Ficetola et al., 2018). However, estimating values of abundance and detection probability is not possible with data from a single site. Nevertheless, it is still possible to estimate the relationships between covariates and detection probability and also to compare the relative importance of these covariates. For this reason, in order to estimate the effect of abiotic factors on detection probability, I used a binomial generalized linear model in a Bayesian framework, specifically written for this analysis (Appendix S1). The following covariates of detectability were included in the model: average temperature during the survey (both quadratic and linear terms), average humidity during the survey, average wind speed during the survey, cumulative precipitation during the survey, cumulative precipitation in the 24 hours before the 
survey; additionally, I included the date, expressed as Julian day, to consider the effect of phenology, and two interactions: date-temperature and date-humidity. Before running the model, I log-transformed precipitation and wind variables to reduce skewness, and then scaled all independent variables of detection with mean of 0 and a standard deviation of 1 (Sokal and Rohlf, 2012). Correlations among independent variables were weak $(|\mathrm{r}|<0.57)$, hence I decided to keep all the predictors in the model. The priors of regression coefficients of the variables related to detection probability were uniform, ranging from -10 to 10 . The model was run with three chains and for 20000 iterations for each chain, discarding the first 10000 iterations as a burn-in. The distribution of posteriors was sampled with a thinning of 10, resulting in 1000 samples for each chain. Parameter convergence was checked both visually and by looking at the Rhat value, which was $<1.01$ for all parameters. Analyses were run in the R environment ( $\mathrm{R}$ Core Team, 2018) using the package R2jags (Su and Yajima, 2015). A script of the model and data used to run the analyses are available in Appendix S1.

\section{Results}

Over the 117 surveys, the number of detected lizards ranged from 0 to 49 (Fig. S2). Julian day showed a negative relationship with average detection probability (Fig. 1), indicating that lizards were easier to detect earlier in the sampling season (Fig. 2a). Detection probability showed a quadratic relationship with temperature (Fig. 1). On average, the highest detection probability was observed at $25.6^{\circ} \mathrm{C}$. The effect of precipitation showed a bimodal pattern. Rainfall during the survey showed a negative relationship with detection probability (Fig.1; Fig. 2b), while rainfall in the 24 hours 
159 before the survey showed an average positive relationship (Fig. 1). This indicates that 160 lizards are less detectable during rains but easier to detect after rainy days. Humidity

161 showed a negative relationship with detection probability, indicating that detection

162 probability was lower during surveys with higher relative humidity (Fig. 1). The

163 average effect of wind was close to zero, with 95\% CIs widely overlapping zero,

164 indicating no effect of wind on detection probability (Fig. 1). The quadratic effect of

165 temperature showed an interaction with Julian day, indicating that the temperature at

166 which detection probability was the highest varied over the sampling season (Fig. 1).

167 For instance, in the early season (mid-April), detection probability was highest at

$16824.3^{\circ} \mathrm{C}$ (Fig. 2c), while later in the season (beginning of August), detection probability

169 was highest at $27.6^{\circ} \mathrm{C}$ (Fig. 2d). On the contrary, Julian day showed a negative

170 interaction with humidity: the negative relationship between humidity and detection

171 probability was not present in the early season (Fig. 2e and 2f).

173 Discussion

Despite being a very common and widespread species, so far, no study assessed the factors driving the detection probability of the common wall lizard. In this study, through Bayesian N-mixture modeling, I showed that the most influential drivers of the detection probability of this species are temperature and phenology, followed by

179 precipitation and humidity. Temperature showed a quadratic relationship with detection probability, indicating that the activity of the common wall lizard is highest between 25 and 28 degrees, decreasing at lower or higher temperatures (Fig. $2 \mathrm{c}$ and $2 \mathrm{~d}$ ). Previous studies found the body temperature of active common wall lizards around $34^{\circ} \mathrm{C}$ (Avery, 
1978; Braña, 1991). This is not in contrast with the results of this study, since the common wall lizard shows an active thermoregulatory behavior, allowing individuals to reach body temperatures higher than the air temperature (Braña, 1991). Obtaining information about the environmental temperatures which maximize the probability of detecting individuals gives useful, practical information to plan the monitoring of this species.

The date of the survey (Julian day) showed a strong negative relationship with detection probability (Fig. 2a). This indicates that, even after accounting for the effect of temperature, phenology plays a significant role in shaping the activity patterns of the common wall lizard. This species usually breeds between March and June (Biaggini et al., 2011), which can explain the higher detectability earlier in the season. However, this relationship might change across life stages or based on other individual characters. For instance study on aggressive behavior showed a contrasting effect of phenology based on lizard color morph (Coladonato et al., 2020). The picture is further complicated by the interaction between date and temperature (Fig. 2c, 2d). Many studies found a shift in body temperature of reptiles over the sampling season (Castilla, Van Damme, and Bauwens, 1999). However, interactions are often not considered in models with detection probability, either because including additional variables is data-demanding or because it produces model convergence issues. Additionally, through the usage of cosinor models, previous studies showed a strong effect of circadian rhythm on hematological variables and protein secretion in this species (Mangiacotti et al., 2019; Sacchi et al., 2020). Implementing cosinor models into N-mixture/occupancy models could be the focus of future research and can potentially improve the precision of estimates of the factors related to detection probability. 

constraints or because it can be related to other biotic factors, such as prey availability (Sun et al., 2001; Bulova, 2002). For example, some species can prefer higher humidity to avoid the risk of dehydration (Daltry et al., 1998), while others might prefer lower humidity to optimize the heat gain (Sun et al., 2001; Spence-Bailey et al., 2010). Here we showed that adult common wall lizards are more detectable when humidity is low (Fig. 2f). However, this relationship might change among sexes or with age (Sannolo, Barroso, and Carretero, 2018; Sannolo et al., 2020). For instance, smaller individuals surface/volume ratio (Sannolo, Barroso, and Carretero, 2018). Further studies are needed to assess if there is intraspecific variation in the factors driving detection probability. Moreover, the presence of a negative interaction between date and humidity suggested that the negative relationship between humidity and detection probability appears only in the late season (Fig. 2e, 2f). A possible explanation is that the preference for low humidity values is overrun by the advantages of being more active during breedings in the early season.

Precipitation can be a key factor influencing the activity of ectotherms (RozenRechels et al., 2019). Rainfall during the survey showed a negative relationship with detection probability (Fig. 1 and 2b), in agreement with the known ecology of the species (Avery, 1978). However, it has to be remarked that only three surveys $(2.5 \%$ of total surveys) were performed during rains (Appendix S1). Contrary to rainfall during the survey, a higher proportion of surveys $(35 \%)$ showed precipitation in the previous 24 hours. Interestingly, cumulative precipitation in the 24 hours before the survey showed a positive relationship with detection probability (Fig. 1). This suggests that 
after rainy days, the activity of this species is enhanced, perhaps to regain the time spent inactive or because invertebrate prey is more abundant after rains (Williams, 1951). In this study, I assessed the effect of abiotic factors on the detection probability of the common wall lizard. Performing a large number of surveys at the same study site allowed me to identify temperature and phenology as the most influential drivers of detection probability, followed by precipitation and humidity. Knowing the factors that affect the probability of detecting an individual of a given species is of primary importance to avoid bias in population size and vital rates estimates (Kéry and Schaub, 2012). Since with a single site, it is not possible to estimate values of abundance and detection probability, future studies can apply this sampling method to multiple sites. Previous capture-mark-recapture studies showed that demographic parameters of the common wall lizard can vary widely at different sites (Gracceva et al., 2008).

Performing counts at multiple sites would allow us to estimate population abundance and to evaluate how microhabitat or landscape characteristics can influence it.

\section{Acknowledgements}

I want to thank D. Delle Monache for the help in writing the model and for the discussion about statistical issues. I am grateful to Federica, Juno, and Zeus for help in planning and performing the fieldwork. The comment of two anonymous reviewers and the associate editor helped improve a first version of the manuscript. 
Avery, R.A. (1978): Activity patterns, thermoregulation and food consumption in two sympatric lizard species (Podarcis muralis and P. sicula) from central Italy. J. Anim. Ecol. 47: 143-158.

Biaggini, M., Bombi, P., Capula, M., Corti, C. (2011): Podarcis muralis (Laurenti, 1768). In: Reptilia. Fauna d'Italia, Vol. XLV, p. 391-401. Corti, C., Capula, M., Luiselli, L., Razzetti, E., Sindaco, R., Eds. Bologna, Italy, Calderini,.

Braña, F. (1991): Summer activity patterns and thermoregulation in the wall lizard, Podarcis muralis. Herpetol. J. 1: 544-549.

Bulova, S.J. (2002): How temperature, humidity, and burrow selection affect evaporative water loss in desert tortoises. J. Therm. Biol. 27: 175-189.

Coladonato, A.J., Mangiacotti, M., Scali, S., Zuffi, M.A.L., Pasquariello, C., Matellini, 268 C., Buratti, S., Battaiola, M., Sacchi, R. (2020): Morph-specific seasonal variation of aggressive behaviour in a polymorphic lizard species. PeerJ 8: 1-16.

Cunningham, H.R., Rissler, L.J., Buckley, L.B., Urban, M.C. (2016): Abiotic and biotic constraints across reptile and amphibian ranges. Ecography (Cop.). 39: 1-8.

Daltry, J.C., Ross, T., Thorpe, R.S., Wüster, W. (1998): Evidence that humidity influences snake activity patterns: A field study of the Malayan pit viper Calloselasma rhodostoma. Ecography (Cop.). 21: 25-34.

Ficetola, G.F., Barzaghi, B., Melotto, A., Muraro, M., Lunghi, E., Canedoli, C., Parrino, E. Lo, Nanni, V., Silva-Rocha, I., Urso, A., Carretero, M.A., Salvi, D., Scali, S., Scarì G., Pennati, R., Andreone, F., Manenti, R. (2018): N-mixture models reliably estimate the abundance of small vertebrates. Sci. Rep. 8: 10357.

279 Ficetola, G.F., Fanell, M., Garizio, L., Falaschi, M., Tenan, S., Ghielmi, S., Laddaga, L., 280 Menegon, M., Delfino, M. (2021): Estimating abundance and habitat suitability in a 281 micro-endemic snake: the Walser viper. Acta Herpetol. in press.

282 Gracceva, G., Bombi, P., Luiselli, L., Bologna, M.A. (2008): Do demographic aspects 283 of neighbouring lizard populations differ? A case study with the common wall lizard, 284 Podarcis muralis. Amphib. Reptil. 29: 443-448.

285 Kellner, K.F., Swihart, R.K. (2014): Accounting for imperfect detection in ecology: A 286 quantitative review. PLoS One 9: e111436.

287 Kéry, M., Schaub, M. (2012): Bayesian Population Analysis using WinBUGS: A 288 Hierarchical Perspective. Academic Press.

289 MacKenzie, D.I., Nichols, J.D., Hines, J.E., Knutson, M.G., Franklin, A.B. (2003):

290 Estimating site occupancy, colonization, and local extinction when a species is detected 
imperfectly. Ecology 84: 2200-2207.

Manenti, R., Falaschi, M., Monache, D.D., Marta, S., Ficetola, G.F. (2020): Networkscale effects of invasive species on spatially-structured amphibian populations. Ecography (Cop.). 43: 119-127.

Mangiacotti, M., Pezzi, S., Fumagalli, M., Coladonato, A.J., D’Ettorre, P., Leroy, C., Bonnet, X., Zuffi, M.A.L., Scali, S., Sacchi, R. (2019): Seasonal Variations in Femoral Gland Secretions Reveals some Unexpected Correlations Between Protein and Lipid Components in a Lacertid Lizard. J. Chem. Ecol. 45: 673-683.

Mazerolle, M.J., Bailey, L.L., Kendall, W.L., Royle, J.A., Converse, S.J., Nichols, J.D. (2007): Making great leaps forward: Accounting for detectability in herpetological field studies. J. Herpetol. 41: 672-689.

Pérez i de Lanuza, G., Carretero, M.A. (2018): Partial divergence in microhabitat use suggests environmental-dependent selection on a colour polymorphic lizard. Behav. Ecol. Sociobiol. 72: 138.

R Core Team (2018): R: A language and environment for statistical computing. R Found. Stat. Comput. Vienna, Austria.

Royle, J.A. (2004): N-Mixture Models for Estimating Population Size from Spatially Replicated Counts. Biometrics 60: 108-115.

Rozen-Rechels, D., Dupoué, A., Lourdais, O., Chamaillé-Jammes, S., Meylan, S., Clobert, J., Galliard, J.F. Le (2019): When water interacts with temperature: Ecological and evolutionary implications of thermo-hydroregulation in terrestrial ectotherms. Ecol. Evol. 9: 10029-10043.

Sacchi, R., Mangiacotti, M., Scali, S., Coladonato, A.J., Pitoni, S., Falaschi, M., Zuffi, M.A.L. (2020): Statistical methodology for the evaluation of leukocyte data in wild reptile populations: A case study with the common wall lizard (Podarcis muralis). PLoS One 15: e0237992.

Sannolo, M., Barroso, F.M., Carretero, M.A. (2018): Physiological differences in preferred temperatures and evaporative water loss rates in two sympatric lacertid species. Zoology 126: 58-64.

Sannolo, M., Civantos, E., Martín, J., Carretero, M.A. (2020): Variation in field body temperature and total evaporative water loss along an environmental gradient in a diurnal ectotherm. J. Zool. 310: 221-231.

Scali, S., Sacchi, R., Falaschi, M., Coladonato, A.J., Pozzi, S., Zuffi, M.A.L., Mangiacotti, M. (2019): Mirrored images but not silicone models trigger aggressive responses in male Common wall lizards. Acta Herpetol. 14: 35-41.

Scali, S., Sacchi, R., Mangiacotti, M., Pupin, F., Gentilli, A., Zucchi, C., Sannolo, M., Pavesi, M., Zuffi, M.A.L. (2016): Does a polymorphic species have a 'polymorphic' diet? A case study from a lacertid lizard. Biol. J. Linn. Soc. 117: 492-502.

Sillero, N., Campos, J., Bonardi, A., Corti, C., Creemers, R., Crochet, P.A., Isailović, J.C., Denoël, M., Ficetola, G.F., Gonçalves, J., Kuzmin, S., Lymberakis, P., Pous, P. De, Rodríguez, A., Sindaco, R., Speybroeck, J., Toxopeus, B., Vieites, D.R., Vences, 
M. (2014): Updated distribution and biogeography of amphibians and reptiles of

333 Europe. Amphibia-Reptilia 35: 1-31.

334 Sokal, R.R., Rohlf, F.J. (2012): Biometry: The Principles and Practices of Statistics in 335 Biological Research. 4th edition. New York, USA, W.H. Freeman and Company.

336 Spence-Bailey, L.M., Nimmo, D.G., Kelly, L.T., Bennett, A.F., Clarke, M.F. (2010):

337 Maximising trapping efficiency in reptile surveys: The role of seasonality, weather

338 conditions and moon phase on capture success. Wildl. Res. 37: 104-115.

339 Su, Y.-S., Yajima, M. (2015): R2jags: Using R to Run 'JAGS'. R package version 0.53407.

341 Sun, L.X., Shine, R., Debi, Z., Zhengren, T. (2001): Biotic and abiotic influences on 342 activity patterns of insular pit-vipers (Gloydius shedaoensis, Viperidae) from north343 eastern China. Biol. Conserv. 97: 387-398.

344 Williams, C.B. (1951): Changes in insect populations in the field in relation to 345 preceding weather conditions. Proc. R. Soc. B Biol. Sci. 138: 130-156.

346 Zamora-Camacho, F.J., Reguera, S., Moreno-Rueda, G., Pleguezuelos, J.M. (2013):

347 Patterns of seasonal activity in a Mediterranean lizard along a 2200m altitudinal 348 gradient. J. Therm. Biol. 38: 64-69. 
Figure captions:

351

352 Figure 1 Density plots of the posterior distribution for the variables related to detection 353 probability. Thick vertical lines represent the average estimated effect for each variable, 354 outer lines represent the $95 \%$ credible interval and shaded areas represent the $80 \%$

355 credible interval. The superscript “2” indicates a quadratic relationship.

356

357 Figure 2 Relationship between detection probability and some of the most influential 358 variables. In each plot, the thick colored line represents the average predicted 359 relationship, while the thin grey lines represent 3000 samples of the posterior 360 distribution (1000 for each chain). a) Relationship between detection probability and 361 Julian day; b) Relationship between detection probability and cumulated precipitation during the 24 before the survey; The interaction between Julian day and temperature is showed in c and d. c) Relationship between detection probability and temperature during the survey, with Julian day fixed at 102 (mid-April); d) Relationship between detection probability and temperature during the survey, with Julian day fixed at 214 (beginning of August). The interaction between Julian day and humidity is showed in e and f. e) Relationship between detection probability and humidity during the survey, with Julian day fixed at 138 (mid-May); d) Relationship between detection probability and humidity during the survey, with Julian day fixed at 214 (beginning of August). 


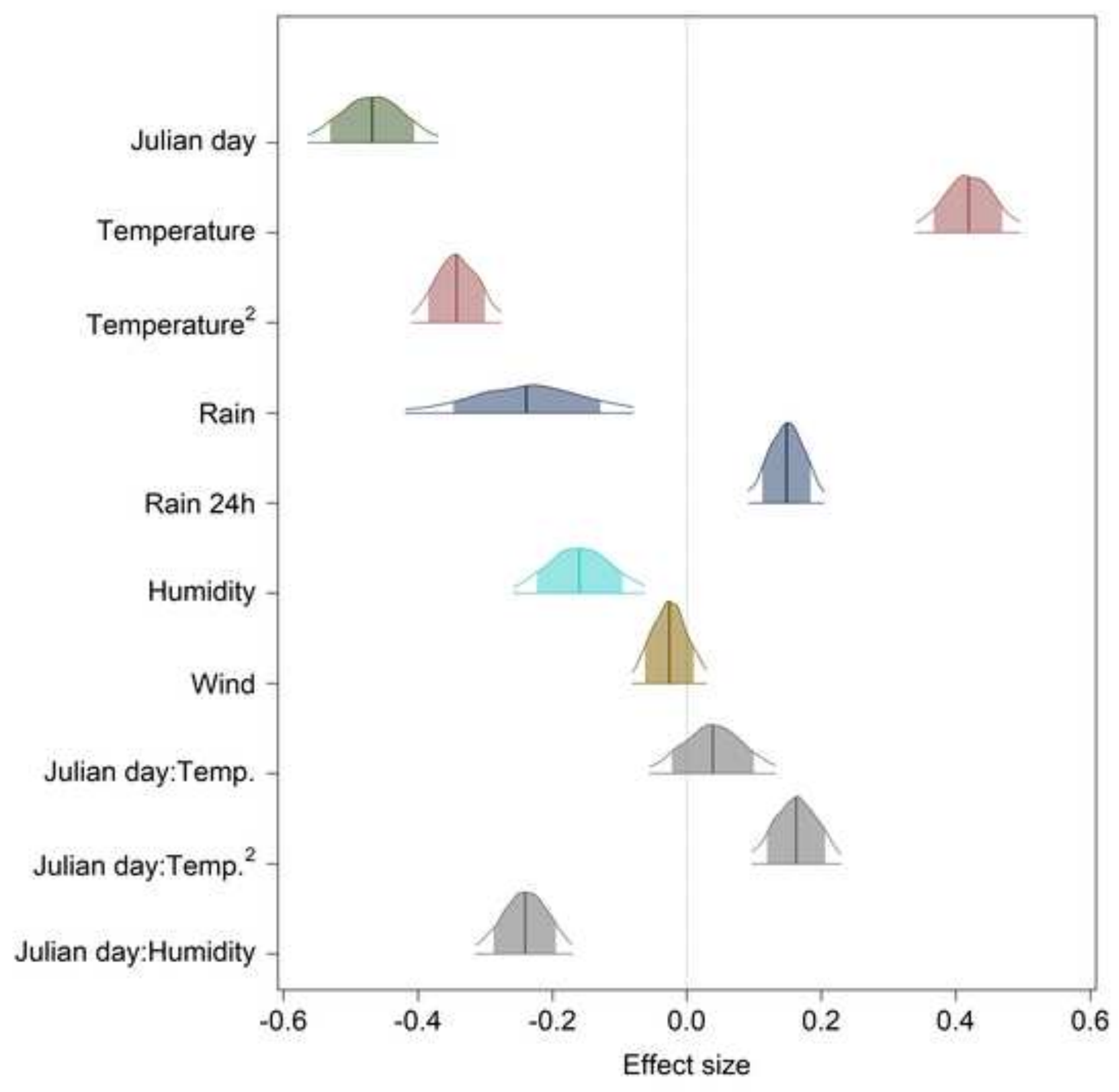



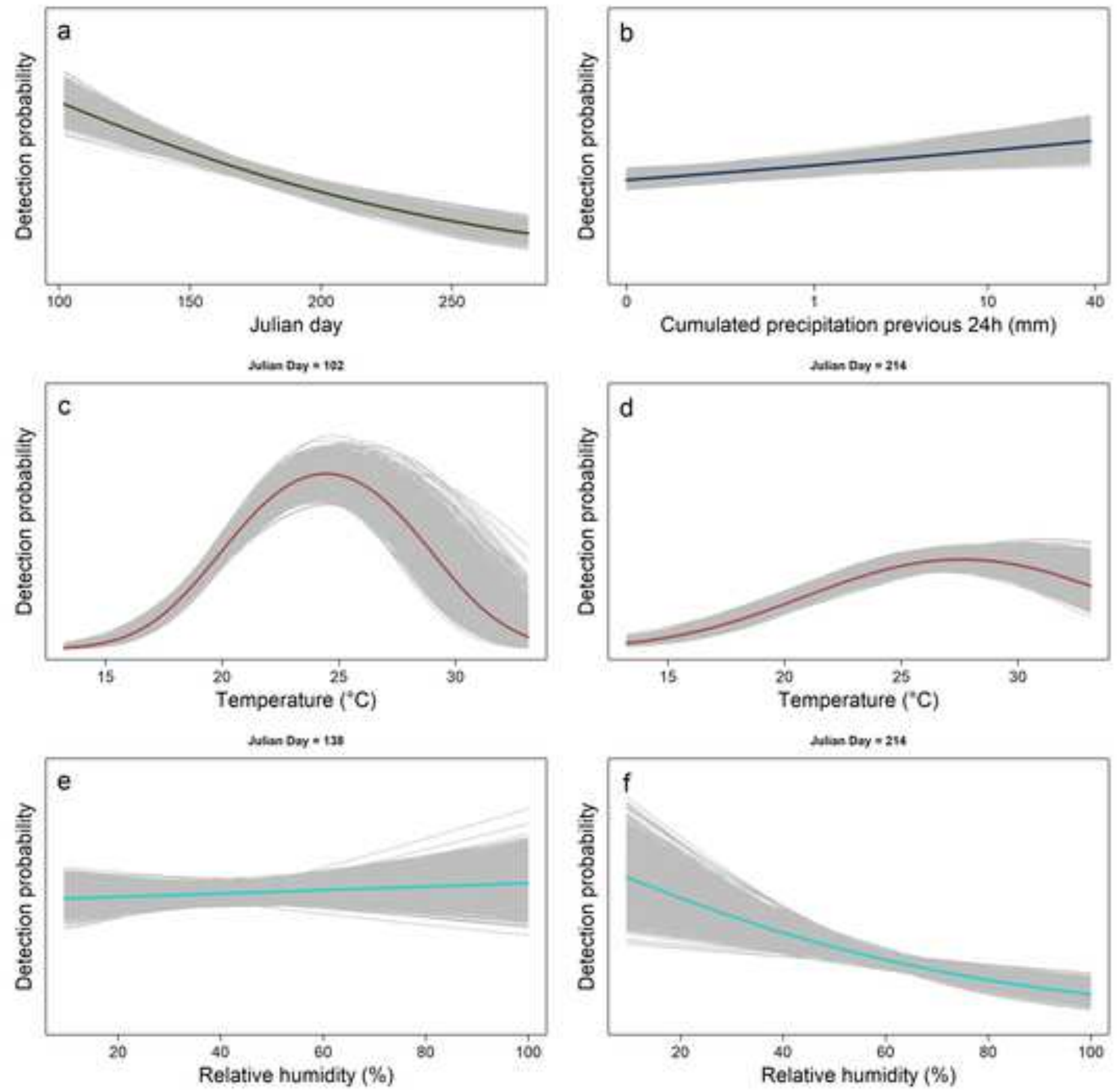
Click here to access/download Supplementary File Supplementary - review1.docx 
Click here to access/download Supplementary File 8a50b95f-0bf5-443e-9465-7717df526bae 
Appendix S2_binomialGLM Podarcis muralis

\section{Click here to access/download \\ Supplementary File Appendix S2_binomialGLM Podarcis muralis.R}

